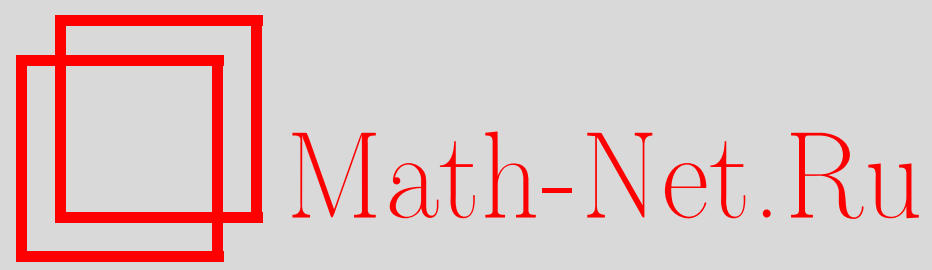

Общероссийский математический портал 
Е. С. Смаилов, О коэффициентах кратных рядов Фурье по периодическим мультипликативным системам Прайса, Матем. заметки, 1996, том 60, выпуск 4, 620-624

DOI: https://doi.org/10.4213/mzm1873 
Использование Общероссийского математического портала MathNet.Ru подразумевает, что вы прочитали и согласны с пользовательским соглашением http://www . mathnet.ru/rus/agreement

Параметры загрузки:

IP : 3.85 .5 .30 
26 апреля 2023 г., 18:11:17

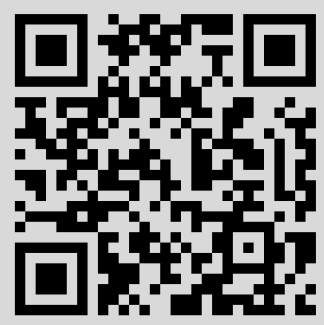




\section{О КОЭФФИЦИЕНТАХ КРАТНЫХ РЯДОВ ФУРЬЕ ПО ПЕРИОДИЧЕСКИМ МУЛЬТИПЛИКАТИВНЫМ СИСТЕМАМ ПРАЙСА}

\section{Е. С. Смаилов}

В данной работе приведены результаты относительно коэффициентов ФурьеПрайса - теоремы типа Харди-Литтлвуда-Пэли и исследована их зависимость от свойства ограниченности образующей последовательности системы Прайса.

Периодические мультипликативные системы Прайса являются достаточно общим классом ортонормальных систем [1], обзор основных результатов по мультипликативным системам на нуль-мерных группах можно найти в $[1],[2]$.

(C) Е.С. Смаилов 
Пусть $\left\{\varphi_{\nu_{i}}^{(i)}\left(x_{i}\right)\right\}_{\nu_{i}=0}^{+\infty}, x_{i} \in G_{i}, i=1, \ldots, n,-$ периодические мультипликативные системы Прайса [1], с образующими последовательностями натуральных чисел $\left\{p_{\nu_{i}}^{(i)}\right\}, i=1, \ldots, n$. Считаем, что

$$
p_{0}^{(i)}=1, \quad p_{\nu_{i}}^{(i)} \geqslant 2 \quad \forall \nu_{i} \in \mathbb{N}
$$

$$
m_{-1}^{(i)}=0, \quad m_{0}^{(i)}=1, \quad m_{N_{i}}^{(i)}=\prod_{\nu_{i}=1}^{N_{i}} p_{\nu_{i}}^{(i)}, \quad i=1,2, \ldots, n .
$$

Пусть $\bar{N}=\left\{N_{1}, \ldots, N_{n}\right\}, N_{i} \in \mathbb{N}, i=1, \ldots, n$;

$$
A_{\bar{N}}=\left\{\bar{k}=\left(k_{1}, \ldots, k_{n}\right) \mid m_{N_{i}}^{(i)} \leqslant k_{i}<m_{N_{i}+1}^{(i)}, k_{i} \in \mathbb{N}, i=1,2, \ldots, n\right\} .
$$

Если $\mathbb{Z}_{n}^{+}=\left\{\bar{\nu}=\left(\nu_{1}, \ldots, \nu_{n}\right) \mid \nu_{i}=0,1,2, \ldots ; i=1, \ldots, n\right\}$, то

$$
\begin{gathered}
\mathbb{Z}_{n}^{+}=\bigcup_{N_{1}=-1}^{\infty} \ldots \bigcup_{N_{n}=-1}^{\infty} A_{\bar{N}}, \\
\left|A_{\bar{N}}\right|=\prod_{i=1}^{n}\left(m_{N_{i}+1}^{(i)}-m_{N_{i}}^{(i)}\right)=\prod_{i=1}^{n} m_{N_{i}}^{(i)}\left(p_{N_{i}+1}^{(i)}-1\right) .
\end{gathered}
$$

Перейдем к изложению основных результатов работы.

ТЕОРемА 1. Пусть $\Phi=\left\{\prod_{i=1}^{n} \varphi_{\nu_{i}}^{(i)}\left(x_{i}\right)\right\}-$ произведение мультипликативных систем Прайса.

$1^{\circ}$. Пусть $2 \leqslant p<+\infty$ и задана таблича чисел $a=\left\{a_{\bar{k}}\right\}_{\bar{k} \in \mathbb{Z}_{n}^{+}}$такая,

$$
D(a)_{p}=\sum_{\bar{\nu} \in \mathbb{Z}_{n}^{+}}^{+\infty} \prod_{i=1}^{n}\left(m_{\nu_{i}+1}^{(i)}\right)^{p / 2-1}\left(\sum_{\bar{k} \in A_{\bar{\nu}}}\left|a_{\bar{k}}\right|^{2}\right)^{p / 2}
$$

сходится, тогда существует функиия $f(x) \in L_{p}\left(G^{n}\right)$, для которой $\left\{a_{\bar{k}}\right\}_{\bar{k} \in \mathbb{Z}_{n}^{+}}$является табличей ее коэффициентов Фурье-Прайса, и при этом

$$
\|f\|_{L_{p}\left(G^{n}\right)} \leqslant B_{p}\left\{\left|a_{0, \ldots, 0}\right|^{p}+D_{p}(a)\right\}^{1 / p},
$$

где $B_{p}>0$ не зависит от таблицы $a, G^{n}=G_{1} \times \cdots \times G_{n}$.

$2^{\circ}$. Eсли $1<p \leqslant 2$ и $f(x) \in L_{p}\left(G^{n}\right)$, тогда для таблици $a=\left\{a_{\bar{k}}\right\}$ eе коэффичиентов Фурье-Прайса имеет место неравенство

$$
B_{p}^{-1}\left\{\left|a_{0, \ldots, 0}\right|^{p}+D_{p}(a)\right\}^{1 / p} \leqslant\|f\|_{L_{p}\left(G^{n}\right)} .
$$

$3^{\circ}$. Если система $\Phi=\left\{\prod_{i=1}^{n} \varphi_{\nu_{i}}^{(i)}\left(x_{i}\right)\right\}-$ произведение мультипликативных систем Прайса с ограниченными образующими последовательностями, то в утверждениях $1^{\circ}$ и $2^{\circ}$ в выражении $D(a)_{p}$ сомножитель $\prod_{i=1}^{n}\left(m_{\nu_{i}+1}^{(i)}\right)^{p / 2-1}$ можсно заменить на $\prod_{i=1}^{n}\left(m_{\nu_{i}}^{(i)}\right)^{p / 2-1}$.

Данная теорема является утверждением типа Харди-Литтлвуда-Пэли. Пункты $1^{\circ}$ и $2^{\circ}$ в настоящей форме для кратных тригонометрических рядов Фурье были впервые установлены В.Н. Темляковым [3]. Доказательство ведется по схеме Харди-Литтлвуда-Пэли [3].

Нам понадобится следующее утверждение, которое доказьвается аналогично лемме $3.1^{\prime}$ из [3]. 
ЛЕмМА. Пусть $1 \leqslant p<r \leqslant+\infty, \Phi=\left\{\prod_{i=1}^{n} \varphi_{\nu_{i}}^{(i)}\left(x_{i}\right)\right\}-$ произведение мультипликативньх систем Прайса. Тогда $\forall \bar{M}=\left(M_{1}, \ldots, M_{n}\right), M_{i} \in \mathbb{N}$, $i=1, \ldots, n$, и заданной табличь чисел $\left\{a_{\bar{\nu}}\right\}_{\bar{\nu} \in \mathbb{Z}_{n}^{+}}$справедливо неравенство

$$
\begin{aligned}
& \left\|\sum_{\nu_{1}=-1}^{M_{1}} \ldots \sum_{\nu_{n}=-1}^{M_{n}}\left(\sum_{\bar{k} \in A_{\bar{\nu}}} a_{\bar{k}} \prod_{i=1}^{n} \varphi_{k_{i}}^{(i)}(\cdot)\right)\right\|_{L_{p}\left(G^{n}\right)} \\
& \geqslant C_{p r}\left\{\sum_{\nu_{1}=-1}^{M_{1}} \ldots \sum_{\nu_{n}=-1}^{M_{n}} \prod_{i=1}^{n}\left(m_{\nu_{i}+1}^{(i)}\right)^{p / r-1}\left\|\sum_{\bar{k} \in A_{\bar{\nu}}} a_{\bar{k}} \prod_{i=1}^{n} \varphi_{k_{i}}^{(i)}(\cdot)\right\|_{L_{r}\left(G^{n}\right)}^{p}\right\}^{1 / p},
\end{aligned}
$$

где $C_{p r}>0$ не зависит от $\bar{M} u\left\{a_{\bar{k}}\right\}$.

ТЕОРема 2. Пусть в произведении $\Phi$ неограничена образующяя последовательность хотя бъ одной системь $\left\{\varphi_{\nu_{i_{0}}}^{\left(i_{0}\right)}\left(x_{i_{0}}\right)\right\}_{\nu_{i_{0}}=0}^{+\infty}$. Tогда в теореме 1 сомнохитель $\prod_{i=1}^{n}\left(m_{\nu_{i}+1}^{(i)}\right)^{p / 2-1}$ из вырахения $D(a)_{p}$ заменить на $\prod_{i=1}^{n}\left(m_{\nu_{i}}^{(i)}\right)^{p / 2-1}$, без нарушения справедливости утвержсдений пунктов $1^{\circ}$ u $2^{\circ}$, нельзя.

ДоказАтЕЛЬство. Достаточно рассмотреть двумерный случай. Пусть система $\left\{\varphi_{\nu_{1}}^{(1)}\left(x_{1}\right)\right\}_{\nu_{1}=0}^{+\infty}$ имеет ограниченную образующую последовательность $\left\{p_{\nu_{1}}^{(1)}\right\}_{\nu_{1}=0}^{+\infty}$, а для системы $\left\{\varphi_{\nu_{2}}^{(2)}\left(x_{2}\right)\right\}_{\nu_{2}=0}^{+\infty}$ образующая последовательность $\left\{p_{\nu_{2}}^{(2)}\right\}_{\nu_{2}=0}^{+\infty}$ неограничена, т.е. $\sup _{\nu} p_{\nu}^{(2)}=+\infty$.

Пусть $2<p<+\infty$. Тогда существует подпоследовательность натуральных чисел $\left\{r_{j}\right\}_{j=1}^{+\infty}$ такая, что $\left(p_{r_{j}+1}^{(2)}\right)^{p / 2-1} \geqslant 2^{j} \forall j \in \mathbb{N}$. Положим

$$
f_{0}\left(x_{1}, x_{2}\right)=\sum_{j=1}^{+\infty}\left(m_{r_{j}}^{(1)} m_{r_{j}+1}^{(2)}\right)^{1 / p-1} \sum_{\bar{k} \in A_{\bar{r}_{j}}} \varphi_{k_{1}}^{(1)}\left(x_{1}\right) \varphi_{k_{2}}^{(2)}\left(x_{2}\right),
$$

где $A_{\bar{r}_{j}}=\left\{\left(k_{1}, k_{2}\right) \mid m_{r_{j}}^{(i)} \leqslant k_{i}<m_{r_{j}+1}^{(i)}, i=1,2\right\}$. Согласно определению множеств $A_{\bar{\nu}}$ и условию теоремы можно утверждать, что

$$
\left|A_{\bar{r}_{j}}\right| \leqslant C_{1} m_{r_{j}}^{(1)} m_{r_{j}+1}^{(2)} \quad \forall j \in \mathbb{N} .
$$

Определение чисел $m_{r_{j}}^{(i)}$ и условия для $p_{\nu_{i}}^{(i)}, i=1,2$, и неравенство $p>2$ позволяют установить, что $f_{0}\left(x_{1}, x_{2}\right) \in L_{2}\left(G^{2}\right)$. Если обозначить через $\left\{b_{k_{1}, k_{2}}\left(f_{0}\right)\right\}$ коэффициенты Фурье-Прайса этой функции, то нетрудно видеть, что ряд

$$
\begin{aligned}
& \sum_{\nu_{1}=0}^{+\infty} \sum_{\nu_{2}=0}^{+\infty}\left(m_{\nu_{1}}^{(1)} m_{\nu_{2}}^{(2)}\right)^{p / 2-1}\left(\sum_{k_{1}=m_{\nu_{1}}^{(1)}}^{m_{\nu_{1}+1}^{(1)}} \sum_{k_{2}=m_{\nu_{2}}^{(2)}}^{m_{\nu_{2}+1}^{(2)}}\left|b_{k_{1}, k_{2}}\left(f_{0}\right)\right|^{2}\right)^{p / 2} \\
& \leqslant C_{p} \sum_{j=1}^{+\infty}\left(m_{r_{j}}^{(2)}\right)^{p / 2-1}\left(m_{r_{j}+1}^{(2)}\right)^{-p / 2+1}=C_{p} \sum_{j=1}^{+\infty}\left(p_{r_{j}+1}^{(2)}\right)^{-p / 2+1} \\
& \leqslant C_{p} \sum_{j=1}^{+\infty} \frac{1}{2^{j}}<+\infty
\end{aligned}
$$


сходится.

Теперь рассмотрим

$$
S_{N}\left(f_{0} ; x_{1}, x_{2}\right)=\sum_{j=1}^{N}\left(m_{r_{j}}^{(1)} m_{r_{j}+1}^{(2)}\right)^{1 / p-1} \sum_{\bar{k} \in A_{\bar{r}_{j}}} \varphi_{k_{1}}^{(1)}\left(x_{1}\right) \varphi_{k_{2}}^{(2)}\left(x_{2}\right) .
$$

Пусть $p<q<+\infty$. Тогда согласно лемме и соотношению

$$
\left\|\sum_{\bar{k} \in A_{\bar{\nu}}} \prod_{i=1}^{2} \varphi_{k_{i}}^{(i)}(\cdot)\right\|_{L_{q}\left(G^{2}\right)} \asymp \prod_{i=1}^{2}\left(m_{\nu_{i}+1}^{(i)}\right)^{1-1 / q}
$$

имеем

$$
\begin{aligned}
\left\|S_{N}\left(f_{0}\right)\right\|_{L_{p}\left(G^{2}\right)}^{p} \geqslant & C_{p q}^{p} \sum_{j=1}^{N}\left(m_{r_{j}+1}^{(1)} m_{r_{j}+1}^{(2)}\right)^{p / q-1} \\
& \times\left(m_{r_{j}}^{(1)} m_{r_{j}+1}^{(2)}\right)^{1-p}\left(m_{r_{j}+1}^{(1)} m_{r_{j}+1}^{(2)}\right)^{p-p / q} \geqslant \bar{C}_{p q}^{p} \sum_{j=1}^{N} 1,
\end{aligned}
$$

так как по условию теоремы $m_{r_{j}}^{(1)} \asymp m_{r_{j}+1}^{(1)}$.

Отсюда следует, что $f_{0}\left(x_{1}, x_{2}\right) \notin L_{p}\left(G^{2}\right)$.

В случае $1<p<2$ контрпример строится по аналогичному принципу.

ЗАмЕчАниЕ. Условия теоремы 1 не улучшаемы в том смысле, что существуют последовательность $\left\{a_{k}\right\}$ в пункте $1^{\circ}$ и функция $f_{0}(x)$ в пункте $2^{\circ}$, для которых условия, а вследствии этого, и утверждения теоремы при данном " $p$ " не выполняются. В то же время, $\forall r: 2<r<p$ в пункте $1^{\circ}, 1<r<p<2$ в пункте $2^{\circ}-$ утверждения теоремы выполнены.

Достаточно рассмотреть одномерный случай. Пусть $1<p<+\infty$. Рассмотрим ряд

$$
\sum_{\nu=1}^{+\infty} m_{\nu+1}^{1 / p-1} \sum_{k=m_{\nu}}^{m_{\nu+1}-1} \varphi_{k}(x)
$$

где $x \in G$. Положим $a_{k}=m_{\nu+1}^{1 / p-1} \forall k \in\left[m_{\nu}, m_{\nu}-1\right] \cap \mathbb{N}, \nu \in \mathbb{N}$.

Пусть сначала $2<p<+\infty$. Тогда $\forall r \in(2, p)$ имеем

$$
D(a)_{r} \leqslant C_{r} \sum_{\nu=1}^{+\infty} m_{\nu+1}^{r / p-1}<+\infty .
$$

Тогда согласно теореме $1 \exists f_{0}(x) \in L_{r}(G)$, для которой ряд (2) является рядом Фурье-Прайса, но

$$
D(a)_{p} \geqslant C_{p}\left\{\sum_{\nu=1}^{N} m_{\nu+1}^{p / 2-1} m_{\nu+1}^{p(1 / p-1)} m_{\nu+1}^{p / 2}\right\}^{1 / p}=C_{p} N^{1 / p} \quad \forall N \in \mathbb{N},
$$

т.е. $D(a)_{p}=+\infty$. Поэтому $f_{0}(x) \notin L_{p}(G)$. В самом деле, для частной суммы $S_{m_{N+1}}\left(f_{0}, x\right)$ ряда $(2)$ с помощью леммы, как и в теореме 2 , устанавливается неравенство

$$
\left\|S_{m_{N+1}}(\cdot)\right\|_{L_{p}(G)} \geqslant C_{p} N^{1 / p} \quad \forall N \in \mathbb{N} .
$$


Следовательн, $f_{0}(x) \notin L_{p}(G)$, хотя, как было показано, $\forall r \in(2, p) f_{0}(x) \in L_{r}(G)$. Пусть теперь $1<p<2$. Тогда $\forall r \in(1, p)$ ряд (2) определяет функцию $f_{0}(x) \in L_{r}(G)$ и согласно пункту $2^{\circ}$ теоремы $1 \quad D(a)_{r}<+\infty$. По лемме

$$
\begin{aligned}
& \left\|S_{m_{N+1}}(\cdot)\right\|_{L_{p}(G)} \\
& \geqslant C_{p}\left\{\sum_{\nu=1}^{N} m_{\nu+1}^{p / 2-1}\left\|m_{\nu+1}^{1 / p-1} \sum_{k=m_{\nu}}^{m_{\nu+1}-1} \varphi_{k}(\cdot)\right\|_{L_{2}(G)}^{p}\right\}^{1 / p} \geqslant C_{p}^{\prime} N^{1 / p} \quad \forall N \in \mathbb{N} .
\end{aligned}
$$

Это означает, что $f_{0}(x) \notin L_{p}(G)$. Поэтому $D_{p}(a)=+\infty$. В самом деле,

$D(a)_{p} \geqslant C_{p}\left\{\sum_{\nu=1}^{N} m_{\nu+1}^{p / 2-1} m_{\nu+1}^{p(1 / p-1)}\left(\sum_{k=m_{\nu}}^{m_{\nu+1}-1} 1\right)^{p / 2}\right\}^{1 / p}=C_{p} N^{1 / p} \forall N \in \mathbb{N}$,

т.е. $D(a)_{p}=+\infty$. В то же время, как уже известно, $\forall r \in(1, p): D_{r}(a)<+\infty$, что и требовалось доказать.

Карагандинский государственный университет им. Е.А. Букетова

\section{СПИСОК ЦИТИРОВАННОЙ ЛИТЕРАТУРЫ}

1. Агаев Г.Н., Виленкин Н.Я., Джафарли Г.М., Рубинштейн А.И. Мультипликативные системы функций и гармонический анализ на нуль-мерных группах. Баку: ЭЛМ, 1984. 2. Голубов Б.И., Ефимов А.В., Скворцов В.А. Ряды и преобразования Уолша. Теория применения. М.: Наука, 1987. 3. Темляков В.Н. Приближение функции с ограниченной смешанной производной // Тр. МИАН. 1986. Т. 178. 